\title{
Staphylococcus aureus: Prevalence among normal healthy individuals
}

\author{
Finney $D,{ }^{1 *}$ Ranganathan $R,{ }^{1}$ Khan AKS, ${ }^{1}$ Shanmugam $J^{2}$ \\ ${ }^{1}$ Department of Microbiology, Gulf Medical University, Ajman, UAE, \\ ${ }^{2}$ Former Professor, Department of Microbiology, Gulf Medical University, Ajman, UAE
}

*Corresponding Author:

Mrs. Devapriya Finney

Department of Microbiology,

Gulf Medical University,

Ajman, UAE

Email id: devapriya31@yahoo.co.in

\section{Citation}

Finney $D$, Ranganathan $R$, Khan AKS, Shanmugam J. Staphylococcus aureus: Prevalence among normal healthy individuals. Nepal Journal of Medical Sciences 2013;2(2):160-4.

\begin{abstract}
Background: To determine the prevalence of Staphylococcus aureus among normal healthy individuals in relation to age, gender and site of isolation.
\end{abstract}

Methods: A cross sectional study was conducted among healthy students and staff volunteers between the age group 15-65 years from Gulf Medical University (GMU) and Gulf Medical College Hospital and Research Centre (GMCH\&RC), Ajman. Aseptically collected nasal and throat swabs were processed for direct Gram stain Microscopy and cultured on appropriate media. Based on the growth on Mannitol Salt Agar and tube coagulase test Staphylococcus aureus and Coagulase negative Staphylococci (CoNS) were grouped.

Results: Of the 127 voulnteers screened 67 were from GMU and 60 from GMCH\&RC. From 49/127 (38.5\%) volunteers 124 Staphylococci isolates were isolated. Of which 62 (50\%) were Staphylococcus aureus and 62 (50\%) were CoNS. Among the 62 Staphylococcus aureus 35 (56.45\%) were from nasal swab and 27 (43.54\%) were from throat swab. Of the 62 CoNS $44(70.96 \%)$ were from nasal swab, $4(6 \%)$ from throat swab and $14(22.58 \%)$ were from both nasal and throat swabs.

Conclusion: The study revealed the asymptomatic inhabitation of Staphyloccus aureus in the nose and throat of healthy individuals. This should be seriously looked into since this in future may lead to carrier status. This alarms the hospital infection control committee to screen students and staff on regular basis to minimize the carrier status to protect the community.

Keywords: Staphylococcus aureus, CoNS- Coagulase negative Staphylococci

\section{Background:}

Staphylococcus aureus remains a versatile and dangerous pathogen in humans. The frequency of both communityacquired and hospital-acquired staphylococcal infections has increased steadily, with little change in overall mortality. Treatment of these infections has become difficult because of the emergence of multidrug-resistant strains. ${ }^{1}$ Colonizing strains may serve as endogenous reservoirs for overt clinical 
infections or may spread to other patients. It is transmitted from person to person by touch, which is an important infection route of nosocomial infections. The nasopharynx is the main ecological niche of the human pathogen Staphylococcus aureus. ${ }^{2}$ Several studies have shown that carrier status has been detected in samples taken from the nasal vestibules. Although Staphylococcus aureus is widely distributed in the human body, including the nose, throat, intestine, skin, etc. the elimination of carriage in the anterior nares, which is the principal reservoir of Staphylococcus aureus, reduces the incidence rate of Staphylococcus aureus infections. ${ }^{3,4}$ The aim of this study is to survey the incidence of Staphylococcus aureus in the nose and throat of healthy adults who are medical students and hospital and college staff members.

\section{Methods:}

Subjects: A total of 127 normal healthy volunteers who were not aware of any illness and not under any medication for the past one month and during the time of sample collection were included in the study. Volunteers were from the Gulf Medical University (GMU) and Gulf Medical College Hospital \& Research Center (GMCHRC) within the age group from 15 years to 65 years. This study was conducted over a one year period. The nasal and throat swabs were collected under aseptic conditions and processed. Samples were collected from the anterior nares and throat of each subject with a cotton swab. The swab was carefully inserted a short distance in each nostril and gently rotated for 5 seconds. For the throat swab collection, the posterior wall of the pharynx and tonsils were swabbed with a sterile cotton swab. A tongue depressor was used, when needed, to depress the tongue.

Isolation and Identification: The swabs were processed within two hours from the time of collection and streaked directly onto Blood agar, Mannitol Salt Agar ${ }^{5}$ and Mac Conkey agar from Hi-media and a direct Gram stained smear had been made. The plates were inspected after 24 hours. If growth seemed to be scanty, the incubation was extended further for another 24 hours. Suspected Staphylococcal colonies such as yellow color Beta hemolytic colonies, nonhemolytic colonies of pale white color on the Blood agar, yellow color colonies on the Mannitol Salt Agar and pale pink color colonies on the Mac Conkey agar were tested by Gram staining and catalase test. The Staphylococcal colonies were tested for coagulase production by the standard tube method with the known positive and negative control strains.

Any degree of clot formation was read as positive. Often the plasma was converted into a stiff gel that remained in place when tube was tilted or inverted, but sometimes clots were seen floating in the fluid. All tubes in which the plasma remains wholly liquid or showed only a flocculent or ropy precipitate were read negative. Out of all volunteers only some individuals were harboring Staphylococcus aureus in either nose or throat or at both sites.

The isolated Staphylococcus aureus were tested for Betalactamase production by the standard Iodometric tube method. ${ }^{6}$ These Beta-lactamase producing Staphylococcus aureus strains were further tested for antibiotic susceptibility testing by the standard Kirby-Bauer disc diffusion method using Muller Hinton agar and antibiotic discs from Himedia.

\section{Results:}

A total of 127 healthy volunteers from GMU (22 staff and 45 medical students) and 60 from GMCHRC were studied, of whom 59 were males and 68 were females. Forty nine participants were found to be positive for Staphylococcus aureus either in the nose or throat, or at both sites.

62 isolates were Staphylococcus aureus and 62 were Coagulase negative Staphylococci (CoNS). Out of the 62 Stapylococcus aureus isolates, 45 were found to be Betalactamase producers. These Beta-lactamase producers were further tested for their antibiotic susceptibility pattern. There was a noticeable $100 \%$ susceptibility for the antibiotics Rifampicin, Vancomycin and Cloxacillin and 100\% resistance towards Penicillin and Ampicillin.

The number of Gulf Medical College Hospital staff participants was approximately the same as that of the GMU staff and students (67 and 60). The male/female ratio gender ratio was 59:68, as shown in Table 1.

Table 1: Categorization of gender ratio among the study population groups

\begin{tabular}{lcrrrrr}
\hline $\begin{array}{l}\text { Study } \\
\text { Group }\end{array}$ & \multicolumn{2}{c}{ GMU Staff } & \multicolumn{2}{c}{$\begin{array}{c}\text { GMU Medical } \\
\text { students }\end{array}$} & \multicolumn{2}{c}{$\begin{array}{c}\text { GMCHRC } \\
\text { Staff }\end{array}$} \\
& No & $\%$ & No & $\%$ & No & $\%$ \\
\hline Male & 14 & 63.6 & 22 & 48.8 & 23 & 38.3 \\
& & & & & & \\
Female & 08 & 36.3 & 23 & 51.1 & 37 & 61.6 \\
\hline
\end{tabular}

The age ranges between 16 and 65 years. The participation rate was highest among 16-20 years and lowest among 5665 years (Table- 2 ) 
Table 2: Age distribution of the study participants

\begin{tabular}{cccccccc}
\hline $\begin{array}{c}\text { Age } \\
\text { (Years) }\end{array}$ & \multicolumn{2}{c}{ GMU Staff } & \multicolumn{2}{c}{$\begin{array}{c}\text { GMU Stu- } \\
\text { dents }\end{array}$} & $\begin{array}{c}\text { GMCH-RC } \\
\text { Staff }\end{array}$ & Total \\
& Male & Female & Male Female & Male & Female & \\
\hline $16-20$ & -- & -- & 16 & 18 & -- & -- & 34 \\
$21-25$ & 4 & 1 & 6 & 5 & 5 & 5 & 26 \\
$26-30$ & -- & 1 & -- & -- & 12 & 17 & 30 \\
$31-35$ & 2 & 2 & -- & -- & 3 & 05 & 12 \\
$36-40$ & 1 & 2 & -- & -- & 1 & 07 & 11 \\
$41-45$ & 2 & 2 & -- & -- & 1 & -- & 5 \\
$46-50$ & 2 & -- & -- & -- & 1 & 2 & 5 \\
$51-55$ & 1 & -- & -- & -- & -- & 1 & 2 \\
$56-60$ & 1 & -- & -- & -- & -- & -- & 1 \\
$61-65$ & 1 & -- & -- & -- & -- & -- & 1 \\
Total & 14 & 8 & 22 & 23 & 23 & 37 & 127 \\
\hline
\end{tabular}

Both the nasal and throat swabs yielded the same percentage of isolation for Staphylococcus aureus and CoNS (62:62). The isolation number of Staphylococcus aureus in the nasal and throat sites was almost closer (33:29). But among the CoNS, isolation was high from the nasal swabs when compared to those from throat swabs (58:04). Nasal colonization was predominantly CoNS (Table 3 ).

Table 3: Staphylococcal isolation verses site of isolation

\begin{tabular}{lcccc}
\hline $\begin{array}{l}\text { Staphylococci } \\
\text { isolated }\end{array}$ & \multicolumn{2}{c}{ Nasal swab } & \multicolumn{2}{c}{ Throat swab } \\
\hline No. & \% & No. & \% \\
\hline CoNS & 33 & 21.2 & 29 & 18.7 \\
\hline
\end{tabular}

The Staphylococcal aureus nasal colonization among the males was noted to be higher compared to the females (male/ female ratio 22:11). On the other hand the nasal colonization of CoNS was high among the females (male/female ratio 15:29) (Table 4).

Table 4: Isolation of S.aureus, CoNS from nasal and throat swabs of different gender

\begin{tabular}{lcccc}
\hline \multicolumn{1}{c}{$\begin{array}{c}\text { Staphylococci } \\
\text { Isolated }\end{array}$} & \multicolumn{2}{c}{ Nasal swab } & \multicolumn{2}{c}{ Throat swab } \\
Males & Females & Males & Females \\
\hline S.aureus & 22 & 11 & 12 & 17 \\
CoNS & 15 & 29 & 3 & 1 \\
\hline
\end{tabular}

The colonization by Staphylococcus aureus was high among the age group 16-25 years and showed a male predominance (Table5).

Table 5: Isolation of Staph.aureus in different ages groups

\begin{tabular}{ccccccccc}
\hline Age in & GMU Staff & \multicolumn{2}{c}{ GMU Students } & \multicolumn{2}{c}{$\begin{array}{c}\text { GMCHRC } \\
\text { Hospital Staff }\end{array}$} & \multicolumn{2}{c}{ Volunteers } \\
years & With S.aureus \\
& Male & Female & Male & Female & Male & Female & No. & $\%$ \\
\hline $16-25$ & 2 & -- & 11 & 5 & 4 & 4 & 26 & 20.4 \\
$26-35$ & -- & 2 & -- & -- & 5 & 5 & 12 & 9.4 \\
$36-45$ & 2 & 2 & -- & -- & 2 & 1 & 7 & 5.5 \\
$46-55$ & 1 & -- & -- & -- & 1 & 1 & 3 & 2.3 \\
$56-65$ & 1 & -- & -- & -- & -- & -- & 1 & 0.78 \\
Total & 6 & 4 & 11 & 5 & 12 & 11 & 49 & 38.5 \\
\hline
\end{tabular}

The Beta-lactamase producing Staphylococcus aureus was $100 \%$ susceptible to Vancomycin, Rifampicin and Cloxacillin and $100 \%$ resistant to Penicillin and Ampicillin. Other antibiotics were also highly susceptible (Table 6).

Table 6: Antibiotic susceptibility pattern of positive Betalactamase S.aureus $(n=45)$

\begin{tabular}{lcccc}
\hline \multirow{2}{*}{ Antibiotic } & \multicolumn{2}{c}{ Sensitive } & \multicolumn{2}{c}{ Resistant } \\
& No. & \% & No. & \% \\
\hline Penicillin & -- & -- & 45 & 100 \\
Ampicillin & -- & -- & 45 & 100 \\
Cloxacillin & 45 & 100 & -- & -- \\
Rifampicin & 45 & 100 & -- & -- \\
Vancomycin & 45 & 100 & -- & -- \\
Gentamicin & 44 & 97.7 & 1 & 2.2 \\
Ciprofloxacin & 44 & 97.7 & 1 & 2.2 \\
Amikacin & 44 & 97.7 & 1 & 2.2 \\
Cefuroxime & 44 & 97.7 & 1 & 2.2 \\
Oxacillin & 43 & 95.5 & 2 & 4.4 \\
Co-trimoxazole & 42 & 93.3 & 3 & 6.6 \\
Erythromycin & 40 & 88.8 & 5 & 11.1 \\
Amoxyclav & 37 & 82.2 & 8 & 17.7 \\
\hline
\end{tabular}

\section{Discussion:}

To study the rate of Staphylococcus aureus inhabitation in the nasal and throat sites a one-year study was done with 127 healthy volunteers in a medical set up. The subjects were in the age group 15-65 years. The screening involved 
the use of nasal and throat swabs.

It was interesting to note in our study sample a female predominance $(61.6 \%)$, as illustrated in Table 1. Sixtytwo Staphylococcus aureus was isolated from the study population with higher number of isolation among male $34 / 62$ (54.8\%) compared to female 28/62 (45.1\%). Nasal isolates were higher in number than the throat isolates, with a nasal:throat ratio of 33:29 (Table 3).

The study yielded five major findings. Firstly, there were healthy asymptomatic individuals harboring Staphylococcus aureus. Secondly, there was a high isolation of Staphylococcus aureus from the nasal swabs. The study done by Tadayuki in $2010^{7}$ in Japan supports our study with the higher incidental rate of nasal colonization compared to throat. Thirdy, the incidence rate of Staphylococcus aureus was high in the 16-25 year olds (43.3\%). Karina et al.'s study on nasal screening among the students at Medical University, Brazil has detected the nasal colonization of Staphylococcus aureus. ${ }^{8}$ Fourthly, an overall male gender predominance in colonization for Staphylococcus aureus (57.6\%) compared to the female $(41.1 \%)$ was observed. Finally, the antibiotic sensitivity pattern of the Betalactamase positive Staphylococcus aureus showed 100\% susceptibility to Vancomycin, Rifampicin and Cloxacillin and $100 \%$ resistance to Penicillin and Ampicillin. There was $82 \%$ susceptibility to Oxacillin. Multidrug resistance was not observed in our study. Graham et al. observed that mecA-negative isolates were sensitive on disc testing to Oxacillin and methicillin. ${ }^{9}$ A comparative susceptibility test methods with mecA gene analysis in Staphylococcus aureus for determining Oxacillin done with the clinical isolates by Cekoska $\mathrm{Z}$ et al. ${ }^{10}$ have detected that the mecA negative strains were Oxacillin susceptible. Since Oxacillin is a semisynthetic drug it will retain its susceptibility for Beta-lactamase producers. ${ }^{11}$ The Beta-lactamase producing Staphylococcus aureus in our study were not multidrug resistant and this could probably be MSSA.

This study showed the prevalence of Staphylococcus aureus colonization among healthy adults, especially among the male. This could lead to the possibility of potential carrier state in due course of time. It is advisable for all medical personnel especially the clinical members to undergo routine screening for the detection of Staphylococcus aureus from the nose and throat. Elimination of carriers will reduce the source of nosocomial infection. By this surveillance method we can also detect and treat the MRSA carriers. Besides the threat of MRSA carriers, MSSA carriers are also to be considered because MSSA may become a serious nosocomial pathogen. ${ }^{12}$ Staphylococcus nasal carrier state can be eliminated by using $2 \%$ mupirocin nasal ointment and washing with chlorhexidine soap. ${ }^{13,14}$

\section{Conclusion:}

From our study we conclude that Staphylococcus aureus can asymptomatically inhabit the nose or throat of an individual. This should be seriously taken into consideration since it could lead to a carrier state in future and attention should be given to all asymptomatic healthy individuals.

There should be a regular quarterly screening surveillance among all the students and staff working in the medical set up especially those in the clinical postings, with strict focus towards the individuals posted in the NICU, neonatal wards/ clinics, ICU, transplantation units, dialysis units, surgical wards etc. Those individuals in whom it is difficult to eradicate the inhabitation of multidrug resistant organisms should have a change of work units so that the patients will be at no risk.

\section{Acknowledgements:}

We thank all the volunteers who came forward to support this study and our Gulf Medical University Management and the Department of Microbiology for the encouragement and support.

\section{Conflict of interest: none declared}

\section{References:}

1. Franklin DL. Staphylococcus aureus Infections. N Engl J Med 1998;339:520-32.

2. Marc B, Christiane W, Christiane G. Regulatory adaptation of Staphylococcus aureus during nasal colonization of humans; Available from: URL: PLoS ONE 5(4): e10040. doi:10.1371/journal.pone.0010040.

3. Christof VE, Karsten B, Konstanze M, et al. Nasal Carriage as a Source of Staphylococcus aureus Bacteremia. N Engl J Med 2001;344:11-6.

4. Emiko U, Shige K, Naomi H, et al. Comparative Characterization of Staphylococcus aureus isolates from throats and noses of healthy volunteers, Jpn. J Infect Dis 2004;57:21-4.

5. Nouwen JL, Ott A, Marjolein FQ, et al. Predicting the Staphylococcus aureus Nasal Carrier State:Derivation and Validation of a "Culture Rule" Clinical Infectious Diseases 2004;39:806-11.

6. Kilic E, Yalinay M. Comparison of Staphylococcal 
Beta-lactamase Detection Methods. J Pharm Sci 2006;31:79-84.

7. Tadayuki I, Yoshio U, Hitomi S, et al. Staphylococcus epidermidis Esp inhibits Staphylococcus aureus biofilm formation and nasal colonization, Nature 2010 May 20;465:346-9.

8. Karina AP, Torres AM, Lourdes BG, et al. Nasal carriage of methicillin-reisistant Staphylococcus aureus in university students. Braz J Infect Dis 2010;14:316-8.

9. Graham JC, Murphy OM, Stewart D, et al. Comparison of PCR detection of mecA with methicillin and oxacillin disc susceptibility testing in CoagulaseNegative Staphylococci, J Antimicrob Chemother 2000;45:111-3.

10. Cekovska Z, Panovski N, Petrovska M. Methicillinresistant Staphylococcus aureus: Comparison of susceptibility test methods with mecA gene analysis for determining oxacillin(Methicillin) resistance in our clinical isolates. Bratisl Lek Listy 2005;106:163-7.

11. Lomaestro BM. What antibiotics covers MethicillinSensitive Staphylococcus aureus (MSSA) infection? Medscape Pharmacists posted 02.12.2009; Available From; http://www.medscape.com/viewarticle/587892

12. David MZ, Boyle-Vavra S, Zychowski Dl, et al. Methicillin-Susceptible Staphylococcus aureus as a predominantly healthcare associated pathogen: A possible reversal of roles? Abvailable from :URL: PloS ONE: Research Article, 13 apr 2011, 10.1371/journal. pone.0018217.

13. Kluymans J, Van Belkum A, Verbrugh H. Nasal carriage of Staphylococcus aureus: Epidemiology, underlying mechanism and associated risks. J Clin Microbiol Rev 1997; 10:505-520.

14. Glare J. Identification and decolonising nasal carriers of $\mathrm{S}$. aureus can reduce infections, $\mathrm{N}$ Engl J Med 2010;352:9-17,75-7. 